Brit. F. vener. Dis. (1976) 52, 94

\title{
The medical manpower situation in the United States in relation to the sexually transmitted diseases
}

\author{
THE LATE BRUCE WEBSTER \\ From the Cornell University Medical College and the New York Hospital, New York
}

There is no question but that the United States has a problem as regards venereal disease. This was responsible for the reappraisal in 1972 of the entire programme by a National Commission (Webster, 1972) appointed by the Secretary of Health, Education and Welfare. The crux of the problem is that 80 per cent. of cases of sexually transmitted diseases are treated privately by general practitioners, most of whom have not received adequate training in the control of these diseases and are not easily able to obtain laboratory facilities for diagnostic procedures. There is therefore a tendency to treat empirically without making an accurate diagnosis.

From the point of view of the patient the scarcity and inaccessibility of free clinics is a difficulty, and many such clinics are staffed by inadequately trained doctors.

What is the answer to this situation? As was pointed out by the National Commission (Webster, 1972) and the Report of the International Travelling Seminar (PAHO, 1974), the first step appears to be the reintroduction of training in the sexually transmitted diseases into our medical schools. To supplement this, for the benefit of those physicians who are already in practice, facilities for graduate seminars at the level of the State and County medical society is needed. The reintroduction and expansion of cooperative clinics between City and State Health Departments and university hospitals connected with medical schools could play a major role in this as illustrated by pilot experiments at the Cornell and Meharry Medical Schools. The National Commission on Venereal Diseases recommended an expansion of this programme.

The cost of treating the sexually transmitted diseases is an important factor. The charges by private physicians and university clinics are of necessity high. The expansion of a system of wellconducted city or state maintained and adequately staffed public clinics, preferably associated with a

Presented at the 28th General Assembly of the IUVDT, Malta, April, 1975

Address for reprints: L. M. Drusin, M. D., New York Hospital, 525 East 68th Street, New York 10021, U.S.A.
University or Voluntary Hospital, would be an inducement for the return of patients to this system. Other countries have demonstrated this to be the case.

The diagnosis and treatment of the sexually transmitted diseases is the responsibility of each State in the United States and is independent of the Federal government. The individual States and Cities have Health Commissioners who appoint administrative chiefs of the venereal diseases services. The Health Departments of the various Cities and States maintain approximately 2,000 clinics scattered throughout the country. However, as stated above, a survey has shown that 80 per cent. of patients are treated by private physicians.

The Federal centre responsible for the control of the venereal diseases is situated in the Center for Disease Control (CDC) in Atlanta, Georgia, and is a part of the Department of Health, Education and Welfare. Block grants and project grants may be made by this Centre to individual states and cities. In addition, they maintain statistics and act in an overall consultant capacity.

The situation, as it exists today, is very different from that 30 years ago when the majority of cases of sexually transmitted diseases were treated in centres maintained by the United States Public Health Service. With the advent of penicillin and the simplification of treatment, there was a tendency for the general practitioner to take over the management of these cases, and today only a few university hospitals and other voluntary health centres maintain clinics for the control of these diseases.

In the pre-penicillin era, syphilis was treated largely by dermatologists in the United States. However, as early as 1925 , two University clinics, Vanderbilt and Johns Hopkins, created clinics as a part of the Department of Internal Medicine for the diagnosis and treatment of syphilis, and these clinics gradually encompassed the other sexually transmitted diseases. One of these clinics was established at Cornell-New York Hospital in 1935. At this time, gonorrhoea was treated largely by the urologists and gynaecologists. 
In 1971 a group of experts, sponsored by WHO and the IUVDT, toured the United States and evaluated the venereal disease control programme as it existed at that time (PAHO, 1974). They pointed out that, in general, there were too few clinics to serve the number of patients. In some large cities no free clinics existed, and as a rule the physical facilities were poor. There were insufficient doctors to deal with the case load ; most were part-time, working on a sessional basis, and many had had little specific training in the diseases they were treating.

Several years ago the teaching of the clinical, epidemiological, and social aspects of the venereal diseases in the medical schools throughout the world was the subject of a cooperative investigation by the WHO and IUVDT. This report pointed out that the teaching of the sexually transmitted diseases in medical schools throughout the United States was everywhere inadequate and in some places nonexistent. This was confirmed by the Report of the International Travelling Seminar (PAHO, 1974):

'The teams visited a number of medical schools throughout the country and, in all of these, teaching about the venereal diseases was inadequate and frequently nonexistent. In some Universities a maximum of 2 hours was set aside for teaching about syphilis, and gonorrhoea was not mentioned. Occasionally, if the teachers were interested, gonorrhoea might be discussed in the urological and gynaecological departments'.

There was no integrated teaching about any of the other sexually transmitted diseases except in two or three medical schools where special programmes existed. It was pointed out that, if more doctors could be persuaded to work in this field and if a greater interest were to be taken in these diseases by the profession as a whole, it was essential that adequate undergraduate teaching, which should include all sexually transmitted diseases, be given to all medical students.

When the Secretary of Health, Education and Welfare appointed the National Commission on the Venereal Diseases in 1971, the commission was charged (1) to outline way to improve among medical students and practising physicians, the knowledge of the clinical management of and public responsibility for venereal diseases; (2) to devise ways to increase the understanding among private physicians of the venereal disease problem; and (3) to bring public health and private medicine into a closer working relationship. In 1972, the report of this commission recommended that:

'The Deans, faculty and committees on curriculum of medical schools be made aware of the importance of including a minimal number of hours in venereology as part of a curriculum and that a faculty member who has a primary interest in venereology be identified to coordinate the teaching efforts of the several clinical departments and the department of preventive medicine to C insure that undergraduates will be introduced to an optimal amount of venereology'.

It was further recommended that such a faculty position be supported in part or totally by a Federal grant of $\$ 20,000$ to $\$ 30,000$ per year. and that liaisons be set up between city and state free clinics and the University clinics in order to provide adequate teaching material for these diseases.

In both the Report of the International Travelling Seminar and that of the National Commission on Venereal Diseases, cognizance was taken of the fact that the great group of private practitioners who were treating 80 per cent. of the cases of the sexually transmitted diseases in the United States had had, during their undergraduate medical education, little or no training in the control of these diseases. The Travelling Seminar pointed out that postgraduate medical education about venereal diseases was virtually nonexistent in the areas in which they visited. They stressed the need for postgraduate courses and training on the subject to be made widely available to all types of doctors.

The National Commission further recommended that:

'The medical organizations oriented to family practice, emergency room practice, and preventive medicine, as well as certain associations of specialists and their Boards of Certification, emphasize the need for education in venereology at the level of graduate training in both university and community hospitals'.

What is the status of venereal disease control in the United States today?

Gonorrhoea, the most frequently reported communicable disease in that country today, continued to increase during 1974 and the reported cases reached a total more than double that of 6 years before. The total of 874,161 cases reported in the 12 -month period ending June 30,1974 , was 64,480 (8 per cent.) higher than the previous year (ASHA, 1975). Although in part these changes in the number of cases reported reflect increased case finding as a result of screening in females, there was also an increase in the number of male cases. It is still important to remember that only approximately one case in ten is reported. The complications of gonorrhoea in women are estimated to cost the nation $\$ 211,000,000$ annually, accounting for approximately 175 thousand hospital admissions.

Localized pilot studies indicate a high reinfection rate among both males and females (ASHA, 1975).

The attack rate of infectious syphilis is in all probability stationary. The role which the high gonorrhoea rate plays in this has to be considered. The diagnosis and treatment of gonorrhoea with its short incubation period may be aborting a certain number of cases of incubating syphilis.

VD funding for the Fiscal year 1975 fared relatively well in the Federal budget recommendations, in that 
continuation of project grants at the same level was recommended. The Health, Education and Welfare (HEW) appropriations bill signed by the President on December 9, 1974, included $\$ 28$ million in VD project grant funds (an increase of 3.2 million over the 24.8 million for the year 1974), and $\$ 4.122$ million for National Institute of Allergy and Infectious Diseases (NIAID) for VD research funds (an increase of 1.185 million over the 2.937 million for 1974). However, these gains have been offset to some extent by the fact that the Center for Disease Control has discontinued its extramural VD research grants. Further inflation and automatic salary increases may well decrease the programme. Therefore, it seems doubtful that, in spite of increased VD appropriations, the total Federal funds for the year 1975 will effectively exceed those for the year 1974 . 'Today's VD Control Problem, 1975', published by the American Social Health Association (1975), discusses this problem in some detail. The National Institute of Health has made a serious effort to promote research in the sexually transmitted diseases in the universities throughout the country, and many important projects are being funded by NIAID. In June, 1975, a Congress on the Biology of the Spirochaetes is being held at the University of Minnesota, and this should stimulate extramural research in this field.
However, the latest volume of 'Today's VD Control Problem, 1975' states that 'the recommendations that a conference be called by HEW to consider how undergraduate medical education and continuing education of physicians may be strengthened has not been carried out and the continued lack of progress in this area makes the problem a critical one'.

That drastic and radical measures must be undertaken to stem the rising tide of the sexually transmitted diseases in the United States is obvious. A few suggestions have been made which, if they could be adopted, should play a major role in attacking the problem.

\section{References}

American Social Health Association (1975) 'Today's VD Control Problem'. A.S.H.A., 1740 Broadway, New York

Fleming, W. L., Brown, W. J., Donohue, J. F., and Branigin, P. (1970) f. Amer. med. Ass., 211, 1827

Pan-American Health Organization (1974) 'Report of the International Travelling Seminar on Venereal Diseases in the United States'. Scientific publication No. 280, Government Printing Office, Washington, D.C.

United States Department of Hzalth, Education AND WeLfare (1972) 'Report of the National Commission on Venereal Diseases', ed. B. Webster, Publication No. 72-8125. Government Printing Office, Washington, D.C. 